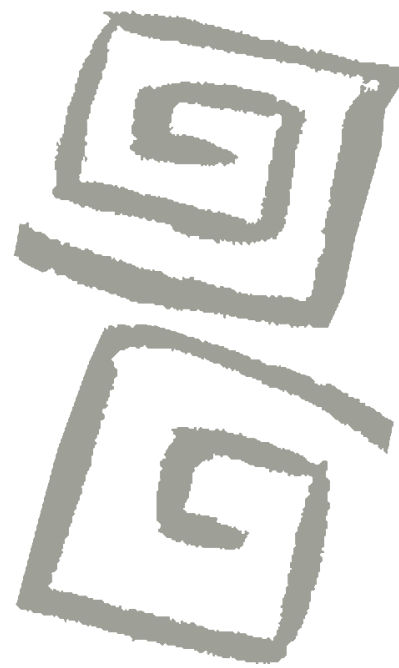

\title{
Aspectos socioepidemiológicos y culturales de la leishmaniasis cutánea: concepciones, actitudes y prácticas en las poblaciones de Tierralta y Valencia, (Córdoba, Colombia)
}

\author{
Socio-epidemiological and cultural aspects of cutaneous \\ leishmaniasis: conceptions, attitudes and practices in the \\ populations of Tierralta and Valencia (Cordoba, Colombia)
}

Sandra Yaneth Patiño-Londoño ${ }^{1}$, Lina Marcela Salazar², Catalina Tovar Acero ${ }^{3}$, Iván Darío Vélez Bernal${ }^{4}$

${ }^{1}$ Antropóloga, Magíster en Antropología.

Coinvestigadora, Programa de Estudio y Control de Enfermedades Tropicales, Universidad de Antioquia, Colombia. $\bowtie$ iD

${ }^{2}$ Microbióloga, Bioanalista, Magíster en Epidemiología. Coinvestigadora, Programa de Estudio y Control de Enfermedades Tropicales, Universidad de Antioquia, Colombia. $\square$ (iD)

${ }^{3}$ Bacterióloga, Laboratorista Clínica, Magíster en Infecciones y Salud en el Trópico. Coinvestigadora, Universidad del Sinú, Colombia. $\bowtie$ iD

${ }^{4}$ Médico, Doctor en Enfermedades Tropicales. Coinvestigador, Programa de Estudio y Control de Enfermedades Tropicales, Universidad de Antioquia, Colombia. $\square$ (iD)
RESUMEN Este artículo se centra en las concepciones, actitudes y prácticas de los pobladores de cuatro veredas en Tierralta y Valencia (Córdoba, Colombia), que han padecido o conocen la leishmaniasis cutánea. Se implementó una metodología mixta que combinó un diseño cualitativo con entrevistas y grupos focales $(n=45)$, y un diseño epidemiológico en el que se aplicó la prueba de Montenegro $(n=251)$, se relevaron casos sospechosos de leishmaniasis cutánea en humanos $(n=5)$ y se realizaron encuestas epidemiológicas $(n=409)$. Entre los resultados se encontró que hay un desconocimiento del vector y, aunque identificaron la Lutzomyia (Alú), no la correlacionaron con la leishmaniasis cutánea. Por otra parte los tratamientos tradicionales caseros son los más usados, lo que provoca el aumento del subregistro de casos. Respecto al personal de salud, se encontraron fallas en el diagnóstico y el tratamiento, lo que refuerza la adherencia a tratamientos caseros. Este escenario nos insta a reflexionar acerca de los retos del sistema de salud respecto a las intervenciones del personal de salud en las comunidades asentadas en zonas endémicas.

PALABRAS CLAVES Leishmaniasis Cutánea; Conocimientos, Actitudes y Práctica en Salud; Epidemiología; Colombia.

\begin{abstract}
This article focuses on the conceptions, attitudes and practices of the inhabitants from four villages (veredas) in Tierralta and Valencia (Cordoba, Colombia), who have suffered from or are aware of the existence of cutaneous leishmaniasis. A mixed methodology was implemented based in a qualitative design using interviews and focus groups $(n=45)$ and an epidemiological design which included applying the Montenegro test $(n=251)$, uncovering suspected cases of cutaneous leishmaniasis in humans, $(n=5)$ and applying epidemiological surveys $(n=409)$. Among the results, a lack of knowledge regarding the vector was found; although respondents could identify the Lutzomyia (known as "alú"), they did not correlate it with cutaneous leishmaniasis. In addition, traditional home treatments were more frequently used, increasing the underrecording of cases. With respect to healthcare personnel, flaws in diagnosis and treatment were found, which reinforces adherence to home treatments. This scenario calls for a reflection upon the challenges of the health care system in relation to the interventions of health personnel in communities situated in endemic areas.
\end{abstract}

KEY WORDS Leishmaniasis Cutaneous; Health Knowledge, Attitudes, Practice; Epidemiology; Colombia. 


\section{INTRODUCCIÓN}

La leishmaniasis es una enfermedad endémica en Colombia, que afecta a las personas que viven hasta los $1.750 \mathrm{~m}$ sobre el nivel del mar, es decir, el $91 \%$ del territorio nacional. Es causada por un parásito del género Leishmania y transmitida a los humanos por medio de un flebotomíneo del género Lutzomyia ${ }^{(1)}$. La leishmaniasis es un grupo de enfermedades con tres manifestaciones clínicas: cutánea, mucocutánea, o visceral, siendo esta última la forma más grave.

La Organización Mundial de la Salud (OMS) considera la leishmaniasis como "la enfermedad que afecta a las poblaciones más pobres del planeta; está asociada a la malnutrición, los desplazamientos, las malas condiciones de vivienda, la debilidad del sistema inmunitario y la falta de recursos"(2), además de ser catalogada como una "enfermedad desatendida".

En Colombia, la Ley 100 de 1993 reglamenta el Sistema General de Seguridad Social y por medio de la Ley 1122 de 2007 se fundamentan los lineamientos de salud pública y se crea el Sistema de Vigilancia SIVIGILA, a través del cual se monitorea la incidencia de las enfermedades de importancia en salud pública y permite ser el soporte para la creación de programas de promoción y prevención. Es por esto que, en 2010, se genera la "Guía para la atención clínica integral de pacientes con leishmaniasis" y, en 2014, el "Protocolo de vigilancia en salud pública de Leishmania", los cuales orientan a escala nacional cómo debe ser el diagnóstico, tratamiento y control de la enfermedad, y cuáles son las directrices para la creación de programas de prevención locales.

Hasta la fecha, en Colombia, la leishmaniasis cutánea se considera un problema de salud pública, ya que afecta a gran parte de la población ${ }^{(3)}$ y los recursos que se emplean para su control y prevención son mínimos. En 2014, se presentaron 10.358 nuevos casos, lo cual representa un $11,6 \%$ más con respecto a 2013. El departamento de Córdoba aportó el $4,5 \%$ de los casos a escala nacional con
$457 \mathrm{y}$, dentro de este departamento, los municipios de Tierralta y Valencia presentaron, según el Sistema Nacional de Monitoreo SIVIGILA, el mayor número de casos: 310 y 31 respectivamente, para el año $2014^{(4)}$. Por el contrario, en 2015 se registró una disminución del $32 \%$ de los casos, en todo el país, por lo que en Tierralta se reportaron solo 127 casos, lo que corresponde al $1,6 \%$ de todos los casos en Colombia ${ }^{(5)}$.

Quienes padecen leishmaniasis cutánea si bien no fallecen por su causa, sufren las consecuencias del estigma social debido a su asociación con el conflicto armado y los contextos de pobreza y vulnerabilidad social, producto del abandono estatal ${ }^{(6,7,8)}$. Este escenario se complejiza con la inexperiencia en el diagnóstico y tratamiento por parte del personal de salud que opera en zonas endémicas, producto del deficiente acompañamiento y capacitación de parte de las autoridades de salud, lo que genera una pérdida de credibilidad en la medicina occidental ${ }^{(9)}$. Como consecuencia de esto, la población en riesgo recurre a otras ofertas terapéuticas que, además de estar más a su alcance, no representan desde sus concepciones ningún tipo de riesgo para su salud, lo que favorece el subregistro de casos y no permite medir la real incidencia de la enfermedad ${ }^{(1,9)}$.

Aunque existan lineamientos a escala nacional sobre el manejo de la enfermedad, no cuentan con directrices claras para el control y prevención. En la mayoría de los municipios las enfermedades transmitidas por vectores se controlan con programas de fumigación, lo que resulta insuficiente para el vector de la leishmaniasis, debido a sus preferencias de hábitat en el peridomicilio y a las condiciones de sus criaderos. Las campañas de tipo educativo y sensibilización frente a la enfermedad son inexistentes, debido a la falta de recursos y el desconocimiento de la enfermedad ${ }^{(9)}$.

En Colombia, son pocos los estudios que se han desarrollado sobre concepciones, actitudes y prácticas respecto de la leishmaniasis cutánea. Estos han permitido identificar algunos aspectos dentro de los sistemas de creencias frente al agente causal, el diagnóstico y el tratamiento, muchos de ellos 
fundamentados en el desconocimiento del origen de la enfermedad, lo que la convierte en causa de estigma para las personas que la padecen $^{(1,10)}$.

Este artículo se enmarca en un proyecto mayor titulado "Diseño de un programa de estudios en infecciones y salud tropical para el departamento de Córdoba" que, entre sus objetivos, se planteó hacer un diagnóstico acerca de las concepciones, actitudes y prácticas de los pobladores que han padecido o conocen la enfermedad, respecto de los signos, el diagnóstico, el tratamiento y los elementos de la cadena epidemiológica. Con este conocimiento se busca generar un diálogo de saberes entre las comunidades y el personal de salud, y con ello crear propuestas de promoción y prevención más enfocadas hacia las necesidades del contexto.

\section{METODOLOGÍA}

Este proyecto fue evaluado y aprobado, en el año 2015, por el Comité de Ética de la Universidad del Sinú (Acta 003). El equipo de investigación estuvo conformado por cinco profesionales: una bacterióloga, un entomólogo, un médico, un veterinario y una antropóloga. Fue una propuesta multidisciplinaria que combinó estrategias de investigación mixta. Se llevó a cabo en el departamento de Córdoba, al norte de Colombia $^{(11)}$, en las veredas de Tuis Tuis y El Loro, en el municipio de Tierralta, que cuenta con 78,972 habitantes, y en Guadual y Mieles, en el municipio de Valencia, que cuenta con 34.654, según el censo de 2005. Estos municipios pertenecen a la subregión del alto Sinú, cercanos a la Serranía de Abibe, lugares propicios para el establecimiento del vector y la transmisión de la leishmaniasis cutánea $^{(12)}$. La temperatura promedio está entre los $28^{\circ}$ y $30^{\circ} \mathrm{C}$. Las principales actividades económicas en la zona se concentran en el cultivo de arroz y la ganadería. Las cuatro veredas están ubicadas a una hora y media aproximadamente de las cabeceras urbanas, en vehículo y por carretera no pavimentada. Aunque cuentan con servicio de transporte público, este opera solo una vez al día. Los animales domésticos y de cría suelen estar sueltos o, en algunos casos, en corrales al lado de la vivienda.

Por tratarse de áreas rurales distantes y con escasos servicios de agua potable y vías de acceso, sus habitantes tienen precarias condiciones de vida, bajos ingresos y acceso deficiente a educación, salud y alimentación, por lo que presentan una mayor vulnerabilidad social. Este escenario se complejizó hace algunos años debido al aumento de desplazamientos forzados, que generó un cambio en los patrones de migración. El mantenimiento de estas condiciones sociales, ecológicas y demográficas coincide con lo reportado por otros autores sobre la epidemiología de la leishmaniasis cutánea, quienes relacionan su ocurrencia con zonas rurales de bajos recursos económicos ${ }^{(13,14,15)}$. Las cuatro veredas fueron escogidas, por un lado, sobre la base de los registros históricos de casos y, por otro, por la seguridad y la facilidad de acceso para el equipo investigador, debido a que en otras zonas de la región que cuentan con registros históricos, no se pudo tener acceso debido a situaciones que alteraron el orden público.

Aunque el diseño del estudio tuvo varias líneas de trabajo, este artículo se centra en dos de ellas: la antropológica y la epidemiológica. Desde la antropología, el diseño cualitativo partió de la conceptualización de las concepciones, como las convicciones propias de las personas, es decir, el conjunto de creencias o principios ideológicos que tienen su arraigo en la interacción del individuo con su cultura; las actitudes, como las disposiciones que le permiten al ser humano actuar selectivamente en sus interacciones sociales y que inciden en la forma de actuar y de comportarse frente a diversas situaciones de la cotidianidad, en este caso frente a una enfermedad o a un tipo de tratamiento, por ejemplo; y, las prácticas, como la expresión de la conducta humana que les permite a los sujetos expresar con acciones sus sentimientos y emociones articulándose con sus actitudes y pensamientos ${ }^{(16,17,18,19)}$. 
Una vez definidas estas categorías iniciales, se dio paso a las entrevistas semiestructuradas. Se comenzó con la búsqueda de participantes que habían padecido leishmaniasis cutánea en los últimos 15 años o que la conocieran, residentes en alguna de las cuatro comunidades durante al menos los últimos cinco años, y que desearan participar voluntariamente. Durante las dos salidas de campo, llevadas a cabo en septiembre y noviembre de 2015, solo encontramos cinco participantes que cumplían con todos los criterios de inclusión (tres mujeres entre los 45 y 57 años de edad y dos hombres de 48 y 52 años, uno de ellos curandero), quienes aceptaron ser entrevistados, luego del consentimiento informado. Este reducido número obedece a que más de la mitad de los pobladores llevan poco tiempo viviendo en la zona.

Debido a la dificultad para hallar actores claves, recurrimos a los grupos focales, otra herramienta metodológica que nos brindó más información. Se realizaron dos con docentes (27 participantes) y uno con personal de salud (13 participantes). En total participaron 45 personas, de las cuales 32 eran mujeres. Además, se aplicaron guías de observación durante las visitas a las comunidades. Tanto las entrevistas como los grupos focales fueron grabados en audio.

Se realizó un muestreo por conveniencia y se llevó hasta el nivel de saturación de la información ${ }^{(20)}$, teniendo en cuenta la disposición de las personas para participar y de la calidad de la información suministrada por ellas. Tanto la guía de entrevistas, como la de grupos focales integraron temáticas acerca de las concepciones, actitudes y prácticas de los pobladores respecto a la leishmaniasis cutánea. Posteriormente, se analizaron las transcripciones, con el apoyo de una matriz en Excel 2010, bajo la perspectiva de categorías emergentes, las cuales fueron cruzadas con las categorías iniciales. Esto permitió identificar encuentros y desencuentros entre lo que "se dice que se hace" y lo que realmente se hace, en relación con el autocuidado frente a la enfermedad. Posteriormente, se describió e interpretó el significado de las categorías.
En cuanto al diseño epidemiológico, este tuvo tres componentes estructurales:

1. Prueba de Montenegro: el objetivo de esta prueba $^{(21,22)}$ es determinar la prevalencia de la enfermedad por medio de una prueba inmunológica. Los datos obtenidos fueron tabulados en Excel 2010 y analizados en el programa Epidat $3.1^{\circledR}$. Se realizó un muestro por conveniencia dentro de las cuatro instituciones educativas, asentadas en las veredas del estudio, se aplicó la prueba a toda la población escolar mayor de 5 años, con la autorización previa de los padres de familia y del menor; además, durante los recorridos casa por casa también se aplicó a los adultos que se encontrarán presentes, previo informe del alcance de la prueba. En los participantes cuyo resultado fue positivo, el equipo de salud se encargó de aclarar dudas sobre la memoria inmunológica.

2. Búsqueda de casos activos de leishmaniasis cutánea en humanos: mediante una estrategia de búsqueda por "bola de nieve", y apoyados en el personal de los centros de salud rurales y líderes comunitarios, se solicitó información sobre personas con lesiones -granos o llagas- de más de 15 días de evolución, compatibles con una úlcera, de bordes levantados y difícil cicatrización, con el fin de realizar una visita domiciliaria ${ }^{(23)}$. Luego se tomaron muestras para examen directo, cultivo y reacción en cadena de la polimerasa (PCR) (24) para sospecha de leishmaniasis cutánea y suero para inmunofluorescencia indirecta (IFI) para sospecha de leishmaniasis mucocutánea ${ }^{(25)}$. En cuanto a los pacientes positivos se informó directamente a la Secretaría de Salud local, la cual se encargó de reportar el caso al SIVIGILA y proporcionar el medicamento (Glucantime ${ }^{\circledR}$ ) bajo supervisión médica.

3. Encuesta epidemiológica: se indagó sobre leishmaniasis cutánea y su relación con características de la vivienda, animales asociados a ella, vegetación y datos históricos de la familia que permitieran identificar factores de riesgo, teniendo como variables 
dependientes la presencia de casos antiguos o nuevos ocurridos en la zona de estudio. El cuestionario fue aplicado a un miembro de la familia, mayor de edad. Los datos obtenidos fueron tabulados en Excel 2010 y analizados en el programa SPSS versión 18.

\section{RESULTADOS}

En este apartado se sintetizan las respuestas de los participantes del estudio (docentes, líderes comunitarios en salud, personal de salud y gente del común) en torno a las concepciones, actitudes y prácticas acerca de la leishmaniasis cutánea, así como los datos arrojados por la encuesta epidemiológica. Los resultados están divididos en cinco bloques temáticos: a) caracterización de la población y presencia de la enfermedad; b) etiología de la enfermedad (concepciones y conocimientos); c) conductas de búsqueda de salud (actitudes y prácticas); d) medidas preventivas (prácticas); y e) leishmaniasis cutánea en el contexto médico rural.

\section{Caracterización de la población y presencia de la enfermedad}

Se aplicaron 409 encuestas epidemiológicas: 146 en El Loro, 61 en Tuis Tuis, 78 en Mieles y 124 en Guadual. En las cuatro veredas el grupo etario más predominante fue de 26 a 60 años, aquí encontramos a las personas laboralmente activas. Respecto al sexo, su distribución fue uniforme en las cuatro zonas. Ningún encuestado refirió haber tenido lesiones activas al momento de responder el cuestionario, solo $35(8,6 \%)$ de ellos comentaron haber tenido lesiones antiguas de 10 a 20 años, 27 (6,6\%) lesiones confirmadas por prueba de Montenegro, y ninguno señaló haberlas adquirido en la zona de estudio (Tabla 1). Es importante acotar que muchos de los pobladores actuales vivieron en veredas vecinas y a raíz de la violencia en la zona fueron desplazados.

Durante la primera y segunda salida de campo se encontraron cuatro casos sospechosos de leishmaniasis cutánea, tres de ellos con lesiones sugestivas en miembros inferiores y superiores, todos con más de seis meses de evolución. Solo en la vereda Guadual se encontró un caso sospechoso de leishmaniasis mucocutánea. Después

Tabla 1. Número de casos antiguos y de lesiones confirmadas de leishmaniasis en los municipios de Tierralta y Valencia, departamento de Córdoba, Colombia, 2015.

\begin{tabular}{|c|c|c|c|c|}
\hline \multirow[t]{2}{*}{ Municipio } & \multirow{2}{*}{$\begin{array}{c}\text { Casos } \\
\text { antiguos }\end{array}$} & \multirow{2}{*}{$\begin{array}{c}\text { Lesiones } \\
\text { confirmadas }\end{array}$} & \multicolumn{2}{|c|}{ Tratamiento } \\
\hline & & & Casero & Antimoniales \\
\hline \multicolumn{5}{|l|}{ Tierralta } \\
\hline El Loro & 14 & 10 & 7 & 3 \\
\hline Tuis Tuis & 8 & 6 & $3^{*}$ & 1 \\
\hline \multicolumn{5}{|l|}{ Valencia } \\
\hline Mieles & 4 & 3 & 1 & 2 \\
\hline Guadual & 9 & 8 & 6 & 2 \\
\hline
\end{tabular}

Fuente: elaboración propia. ${ }^{*}$ No todas las personas recordaban qué tipo de tratamiento recibieron para su enfermedad. 
del análisis de las muestras, solo uno de los pacientes fue positivo para leishmaniasis cutánea, pero la adquirió en un lugar diferente a la zona de estudio.

En las cuatro veredas se realizaron un total de 251 pruebas de Montenegro (146 a menores de 18 años y 105 a adultos). Al determinar la prevalencia de la infección entre las personas a las que se les aplicó la prueba, encontramos que la vereda El Loro tenía la prevalencia más alta $(27,3 \%$ en adultos y $15,4 \%$ en niños), aun así, todos los adultos con resultados positivos manifestaron haber tenido las lesiones antes de habitar la zona, en los menores no se pudo establecer esta relación debido a que muchos de ellos no eran conscientes de la enfermedad o de haberla padecido con antelación. Esto se presentó también en las demás veredas, (Tabla 2). Cuando se analiza la población infantil encontramos un panorama similar a la población adulta. Se destaca la falta de casos positivos en Tuis Tuis, lo que sugiere que la transmisión de la enfermedad no se realiza en el domicilio o no es cercana al casco urbano de la vereda (Tabla 2).
Debido a que solo se presentó un caso activo de leishmaniasis cutánea y que las personas que tuvieron la enfermedad referían haberla adquirido fuera de la zona de estudio o antes de habitar sus actuales residencias, no se pudo realizar un análisis para tratar de determinar factores de riesgo en la comunidad que predispongan a adquirir la enfermedad.

\section{Etiología de la leishmaniasis cutánea}

La leishmaniasis cutánea forma parte de la vida cotidiana de los habitantes de estas cuatro veredas. Allí la enfermedad es conocida como leishmaniasis, "picadura de pito" o simplemente "pito". A pesar de encontrar pocos pacientes en la zona, las personas tienen una representación social de la enfermedad, puesto que al menos un miembro de la familia o un vecino la han padecido.

Todos los entrevistados coincidieron en señalar que la leishmaniasis cutánea no es una enfermedad contagiosa. De hecho, no se encontró relación entre esta y el acceso al trabajo o a la educación, puesto que no se la

Tabla 2. Prevalencia de la enfermedad en niños y adultos de una muestra de la población general. Municipios de Tierralta y Valencia, departamento de Córdoba, Colombia, 2015.

\begin{tabular}{|c|c|c|c|c|c|c|}
\hline \multirow[t]{2}{*}{ Municipio } & \multicolumn{2}{|c|}{$\begin{array}{l}\text { Prueba de } \\
\text { Montenegro } \\
(n=251)\end{array}$} & \multicolumn{2}{|c|}{$\begin{array}{c}\text { Total } \\
\text { positivos } \\
(n=22)\end{array}$} & \multicolumn{2}{|c|}{$\begin{array}{c}\text { Prevalencia } \\
\text { de la muestra }\end{array}$} \\
\hline & $\begin{array}{l}\text { Adultos } \\
\text { (n) }\end{array}$ & $\begin{array}{c}\text { Niños } \\
\text { (n) }\end{array}$ & $\begin{array}{l}\text { Adultos } \\
\text { (n) }\end{array}$ & $\begin{array}{c}\text { Niños } \\
\text { (n) }\end{array}$ & $\begin{array}{l}\text { Adultos } \\
\text { (\%) }\end{array}$ & $\begin{array}{c}\text { Niños } \\
(\%)\end{array}$ \\
\hline \multicolumn{7}{|l|}{ Tierralta } \\
\hline El Loro & 22 & 26 & 6 & 4 & 27,3 & 15,4 \\
\hline Tuis Tuis & 13 & 29 & 3 & 0 & 23,1 & 0,0 \\
\hline \multicolumn{7}{|l|}{ Valencia } \\
\hline Mieles & 28 & 48 & 2 & 2 & 7,1 & 4,2 \\
\hline Guadual & 42 & 43 & 2 & 3 & 4,8 & 7,0 \\
\hline
\end{tabular}

Fuente: elaboración propia. 
considera una causa para perder un empleo o no poder acceder a uno. Respecto al lugar y tipo de cicatriz, el $70 \%$ coincide al señalar que los niños y las mujeres que tienen lesiones en rostro y cuello inspiran sentimientos de lástima, pero no de rechazo. En los hombres se han "naturalizado" estas lesiones, producto de la frecuente interacción de estos en el monte debido a actividades laborales como cultivos, extracción de recursos, vigilancia o seguridad. En este caso, no se encontraron explicaciones mágico-religiosas o mitológicas para la leishmaniasis cutánea.

Hay amigos míos con leishmaniasis y eso más que todo les da a personas que viven en el trópico, dentro de la selva, especialmente a los militares. (Docente, hombre, grupo focal, 2015).

Cuando en la encuesta epidemiológica se indagó sobre el reconocimiento de la enfermedad, la mitad de la población relata que saben reconocer las lesiones generadas por el parásito, aunque muy pocos saben la forma de transmisión ni el vector involucrado (Tabla 3). En esa misma línea, algunos participantes de los grupos focales se valieron de sus propias cicatrices para explicar la lesión, mientras que otros recurrieron a la imagen de una moneda para describir la forma típica de la leishmaniasis cutánea.

A excepción del personal de salud, dos explicaciones centrales sobre la causa de la leishmaniasis cutánea fueron expuestas por los participantes; las cuales fueron categorizadas como de origen vectorial y no vectorial.

\section{Origen vectorial de la leishmaniasis cutánea}

Las explicaciones de origen vectorial fueron menos frecuentes $(15 \%)$, que las no vectoriales. A los participantes que declararon que un "mosquito" transmite la leishmaniasis cutánea se les pidió una descripción y desde su narrativa emergieron nombres locales como: "pito", "ya te veo", "más te veo", "pájaro" y "pájaro malo". Hombres y mujeres coinciden al asegurar que estos insectos viven en el monte o la selva, lejos de las viviendas, en lugares húmedos, especialmente donde hay agua estancada (asociación con Anopheles, también presente en la zona). Además de estas características, el $12 \%$ de los participantes consideran que el mosquito "pica y deja los huevos dentro de los poros abiertos, cuando la piel está sudada", otro $15 \%$ cree que el mosquito "después de picar, orina y deja una llaga".

Tabla 3. Reconocimiento del vector y de la enfermedad por parte de los participantes de la encuesta epidemiológica. Municipios de Tierralta y Valencia, departamento de Córdoba, Colombia, 2015 (n=409).

\begin{tabular}{|c|c|c|c|c|c|c|c|c|}
\hline \multirow[t]{3}{*}{ Municipio } & \multicolumn{4}{|c|}{ Reconoce al vector } & \multicolumn{4}{|c|}{ Reconoce la enfermedad } \\
\hline & \multicolumn{2}{|c|}{ Sí } & \multicolumn{2}{|c|}{ No } & \multicolumn{2}{|c|}{ Sí } & \multicolumn{2}{|c|}{ No } \\
\hline & $\mathrm{n}$ & $\%$ & $\mathrm{n}$ & $\%$ & $\mathrm{n}$ & $\%$ & $\mathrm{n}$ & $\%$ \\
\hline \multicolumn{9}{|l|}{ Tierralta } \\
\hline El Loro $(n=146)$ & 6 & 4,1 & 140 & 95,9 & 123 & 50,0 & 123 & 50,0 \\
\hline Tuis Tuis (n=61) & 17 & 27,9 & 44 & 72,1 & 41 & 67,2 & 20 & 32,8 \\
\hline \multicolumn{9}{|l|}{ Valencia } \\
\hline Mieles ( $\mathrm{n}=78)$ & 27 & 34,6 & 51 & 65,4 & 43 & 55,1 & 35 & 44,9 \\
\hline Guadual $(n=124)$ & 7 & 5,6 & 117 & 94,4 & 63 & 50,8 & 61 & 49,2 \\
\hline
\end{tabular}

Fuente: elaboración propia. 
La picadura de pito dizque es producida por un mosquito que tiene el nombre de pito, vulgarmente, porque científicamente no sé, él tiene contacto con la persona y le deja uno o varios huevos y se empiezan a reproducir creando una infección bastante drástica. (Docente, hombre, grupo focal, 2015).

El nombre de "pito" es muy común en varias zonas de Colombia con presencia de leishmaniasis cutánea, y aunque este nombre está asociado a los triatómicos (vectores de la enfermedad de Chagas), en las comunidades lo emplean indistintamente; por un lado, por el desconocimiento general que tienen de las enfermedades transmitidas por vectores $y$, por otro, como producto de las estrategias que emplean las personas para tratar de explicar lo que acontece en su cuerpo a partir de la información que han recibido, de diferentes fuentes, a lo largo de su vida.

Ahora bien, en este contexto la Lutzomyia es conocida como Alú, insecto que físicamente "es chiquitico, tiene las alitas paraditas, es como blanquito, puya durísimo el condenado y revolotea entre las piernas" (Mujer, docente, grupo focal). Sin embargo, ningún participante halló una correlación entre este flebotomino y la leishmaniasis cutánea.

Se observó que la percepción de severidad de la enfermedad depende en gran medida del sexo del mosquito que picó al afectado, es decir, la cantidad de granos o Ilagas, su extensión y características clínicas están directamente relacionadas con la creencia de que la hembra es quien deja múltiples lesiones y "come la piel y la carne de la superficie, se va abriendo poco a poco, llenando el cuerpo si no se trata", en cambio, el macho solo deja una lesión de gran extensión e "hincha y come la piel y la carne hacia adentro".

En cuanto al "ya te veo", encontramos una relación entre el grado de infección de la lesión y el poder transformador de la palabra. En este sistema de creencias se considera que el grano o llaga puede aumentar su tamaño si el afectado menciona el nombre del mosquito en voz alta, reiteradamente. Tal como lo narra una docente: "¡Ay!, te picó el 'ya te veo', no digas qué fue lo que te picó, porque entre más lo digas, más se crece la herida". Esta creencia es menos popular.

Ahondando en las concepciones sobre este vector, tres de los entrevistados que mencionaron al mosquito, lo hicieron porque "es lo que se dice por ahí"; sin embargo, dudan debido a la gran cantidad de insectos con los que conviven y, desde su creencia, explican: "de ser cierto lo del mosquito, todos tendríamos 'picadura de pito' todo el tiempo".

\section{Origen no vectorial de la leishmaniasis cutánea}

Las explicaciones de origen no vectorial de la leishmaniasis cutánea fueron las más frecuentes $(85 \%)$. A excepción del personal de salud, la gran mayoría de los participantes creen que la leishmaniasis cutánea es consecuencia de una inadecuada práctica corporal, como "una cicatriz que se rasca e infecta", o por la interacción del hombre con la naturaleza, como es el caso de "un bejuco de monte que quema o pica al roce". Esta idea del bejuco es muy popular en la costa pacífica colombiana, donde se lo describe como un animal pequeño en forma de hilo, que vive en el bosque sobre ramas o tron $\cos ^{(17)}$. también puede ser causada por algo externo y desconocido, como "algo que inyecta y no se deja ver" o, básicamente, es la respuesta de un cuerpo débil y agotado al que le faltan vitaminas.

\section{Conducta de búsqueda de salud}

Se refiere a la elección del tipo de medicina y al tipo de sistema de asistencia médica al que usualmente recurren las personas afectadas. El análisis cualitativo nos permitió identificar cuatro de ellos: 1) tratamientos tradicionales caseros, 2) tratamientos de curanderos y herbolarios tradicionales, 3) tratamiento biomédico y 4) tratamientos mixtos. En cuanto a la curación de orden místico-religioso, no fue mencionada. 


\section{Tratamientos tradicionales caseros}

Este tipo de tratamiento es más usado que el biomédico; de hecho, 22 de los participantes han recurrido a él, tanto hombres como mujeres. Como expresa una de las entrevistadas:

...yo he oído que [se cura] con unas inyecciones [risas], pero no las usé, con remedios caseros me curé, me echaba de todo, de todo lo que me decían me echaba. (Mujer, 34 años, vereda Tuis Tuis)

Los afectados recurrieron principalmente a terapias térmicas acompañadas de limpiezas sobre la lesión. En numerosos casos los tratamientos incluyeron drogas farmacéuticas, especialmente antibióticos, petroquímicos y productos veterinarios. Casi siempre son los familiares, principalmente las mujeres, quienes se encargan de suministrarlos. Este es el listado de tratamientos tradicionales que fueron referenciados: i) productos que se aplican directamente sobre la lesión (parafina caliente, aceite de motor, disolvente, thinner, Isodine con limón, gentamicina, ampicilina, caparazón de tortuga morrocoy tostado y molido, palo de yuca en polvo, rodajas frescas de tomate maduro). ii) Procedimientos térmicos sobre la lesión (apoyar una cuchara metálica caliente -al rojo vivo- sobre la llaga o ubicar la llama de una vela frente a la llaga; lo más cercano posible).

\section{Tratamientos de curanderos y herbolarios tradicionales}

En la vereda El Loro encontramos un curandero de culebra y "pájaro" (nombre local para leishmaniasis cutánea), quien tiene varios tratamientos terapéuticos basados en plantas medicinales de la zona. Él es consultado por pacientes de su vereda y de zonas aledañas. Para algunos, su tratamiento es efectivo; sin embargo, otros pacientes terminan recurriendo al tratamiento médico occidental por no encontrar los resultados esperados. Entre los participantes, solo tres hombres habían recurrido a este tipo de tratamiento. En la siguiente narración detallamos el paso a paso del tratamiento del "pájaro":

La curación del pájaro tiene tres curas, por ejemplo, yo le hago la limpieza con Isodine con alcanfor para evitar la infección, luego le echo el Caladril con el Calomed, son dos cremas que se venden en las farmacias, si él se seca con esas dos curitas que le echo lo dejo, porque esa es la cura de él. Si no yo le pongo este palito que se llama "hombre solo" y ya, lo rallo y se lo echo. Si no le sirve, porque eso se ve el efecto enseguida, entonces yo le echo "contra", una planta, a esta contra yo la rallo y la echo aquí [muestra un recipiente], esto es para tomar, lo otro lo tomo con ron, yo la rallo y le doy un poco a la persona con un poquito de ron y varias de estas matas: la "baldivia" y el "verrugoso", con esas tres plantas y el contra yo curo, usted lo ve enseguida, porque eso seca el bordito y ya la cura uno de eso, con tres o cuatro curas. (Hombre, 47 años, vereda El Loro, curandero).

\section{Tratamiento biomédico}

Este tipo de tratamiento es el más temido por los pobladores, ya que hay una percepción negativa generalizada frente al tratamiento con Glucantime ${ }^{\circledR}$, no solo por el dolor ocasionado por las inyecciones o la fobia a las agujas, sino por sus efectos secundarios; aspecto que es recurrente en otras zonas del país y de la región ${ }^{(18)}$. Seis de las entrevistadas habían recurrido a este tratamiento, en cambio, ninguno de los hombres lo había hecho, optaban por automedicarse. Esto podría deberse, en parte, a los mitos en torno al Glucantime ${ }^{\circledR}$, como la esterilidad masculina o la abstinencia sexual prolongada, por ejemplo.

Usualmente, los afectados recurren a este tratamiento como última opción, después de peregrinar entre varios sistemas de salud "menos dañinos" desde su percepción, pero poco efectivos. Cuando recurren al centro de 
salud, Ilegan con lesiones infectadas, de gran tamaño e incluso con daños extra en la piel, debido a los productos químicos que emplean para combatir la enfermedad. Es común que aquellos que acceden a este tratamiento y se curan no vuelven a recurrir a otros sistemas terapéuticos. Sin embargo, también es común que algunos de los que inician el tratamiento no lo culminen puesto que, para ellos, "termina siendo peor la cura que la enfermedad". El paciente lo interrumpe a partir de su percepción de bienestar y a la creencia de curación relacionada con lo húmedo y lo seco. Mientras esté la llaga (húmedo) hay presencia de la enfermedad, pero al secarse, se evidencia la eficacia del tratamiento y, por ende, la recuperación de la salud.

En el hospital sí me hicieron la prueba y al día siguiente fui y correcto era eso; me mandaron las inyecciones y no me las coloqué todas, cuando eso yo estaba más flaca, me mandaron 42 y me coloqué apenas 16, porque yo vi que eso ya se me secó y uno poniéndose eso todos los días, eso es duro, esas inyecciones duelen, yo ya vi que eso se me curó y ya. (Mujer, 36 años, vereda El Loro, docente de preescolar)

Esta creencia es reforzada con ciertas prácticas del personal de salud, por ejemplo, la falta de seguimiento de los casos, la interrupción del tratamiento debido a que la lesión a simple vista "se ve bien", administración inapropiada del tratamiento de acuerdo con las guías internacionales, entre otras.

Además de lo mencionado, entre las razones que llevan a los pobladores a elegir otro tipo de tratamiento encontramos: la lejanía entre los caseríos y los centros de salud, las historias de terceros respecto a sus malas experiencias con el tratamiento, la escasez del medicamento en algunas temporadas del año y la creencia de que el tratamiento solo se brinda en el batallón del Ejército.

Otros dicen no, eso hay que hablar con el batallón, que el batallón es quien tiene unas ampolletas y eso es lo que le sirve a la persona, tiene que inyectarse no sé cuántas veces y entonces eso le va a curar eso. (Mujer, 52 años, líder comunitaria en salud, vereda Tuis Tuis)

\section{Tratamientos mixtos}

Son concebidos como otro tipo de práctica curativa adicional para enfrentar la enfermedad, es decir, tienen la función de mejorar el tratamiento biomédico. Las "hierbas medicinales" en bebidas o emplastos o líquidos desinfectantes sobre la lesión, fueron algunos de los productos automedicados por las mujeres mientras estaban bajo tratamiento biomédico. Catorce participantes recurrieron a este tratamiento, tanto las amas de casa como las docentes.

\section{Medidas preventivas contra la leishmaniasis cutánea}

Se observó que las medidas preventivas contra la leishmaniasis cutánea, tal como fueron reportadas por los pobladores, estaban más relacionadas con su experiencia con las medidas de prevención contra la malaria. Estas medidas fueron diferenciadas en tres grupos.

\section{Medidas de protección personal}

Este tipo de medidas no tienen una correlación con la leishmaniasis cutánea, sino que obedecen al autocuidado desencadenado a partir de las campañas de erradicación de la malaria. Los individuos podían alcanzar cierta protección mediante el uso del toldillo, el cual fue brindado a todas las familias por el proyecto Malaria Colombia. Sin embargo, aunque la gran mayoría de pobladores cuentan con varios toldillos en su vivienda y dicen "usarlo todas las noches", muchos de ellos en la práctica prefieren otras medidas como el uso del ventilador, mientras los toldillos reposan doblados dentro un armario, hasta que la "mosquitera se alborota" y los vuelven a usar. 
Primero los estaba usando, ya después como que me fastidié, los lavé y no los he colocado, pero cuando veo que hay abundancia ahí sí los coloco. Yo no sé si es la creencia o me da como una asfixia o qué, pero siento incomodidad, jes que este toldo me está incomodando! [risas]. (Mujer, 36 años, vereda El Loro, docente de preescolar)

Es frecuente el uso de ventiladores eléctricos durante el día y la noche, ya que en la zona la electricidad es subsidiada y las familias pueden disfrutar de este servicio las 24 horas sin ningún costo, convirtiéndose en una medida que además de mantenerlos frescos, los protege de los vectores, medida que es muy popular en otros países ${ }^{(17)}$. Ninguno de los entrevistados mencionó el uso de repelentes comerciales o de fabricación casera.

\section{Medidas de protección ambiental}

La medida más empleada entre los pobladores es la producción de humo casero para controlar los vectores. Las mujeres, principalmente, son quienes hacen recorridos dentro de la casa valiéndose de recipientes en los que queman cáscaras secas de naranja y limón. Las fumigaciones extensivas no son frecuentes en la zona, así como otras medidas oficiales.

\section{Medidas de compromiso con la comunidad}

Las entidades locales de salud no realizan campañas educativas enfocadas al control de vectores en las zonas rurales. Las pocas que se han Ilevado a cabo han sido lideradas por organizaciones no gubernamentales internacionales y se han centrado en la malaria; mientras que otras enfermedades endémicas como la leishmaniasis cutánea han sido desatendidas. Este escenario se complejiza debido al desconocimiento que tienen los pobladores sobre esta enfermedad.

Organizaciones como "Malaria Colombia", financiado por el Fondo Mundial, que trabajó en la zona entre el 2012 y 2015, han sido exitosas porque han brindado medicamentos y toldos en cada vivienda, han capacitado a líderes locales en salud en temas relacionados con la prevención, diagnóstico y tratamiento de la malaria, para que ellos mismos se encargaran de ejecutar prácticas de cuidado con sus poblaciones.

\section{La leishmaniasis en el contexto médico rural}

A partir de los resultados arrojados durante la sesión de grupo focal con el personal de salud de los municipios de Tierralta y Valencia, se evidenció que los médicos y el personal de enfermería y laboratorio están familiarizados con la manifestación clínica de la leishmaniasis cutánea y consideran que es una enfermedad del área rural. Sin embargo, se identificaron vacíos respecto al diagnóstico, así como imprecisiones en el tratamiento. Se detectó como una dificultad el hecho de que en los centros de salud rurales no se presta el servicio de diagnóstico de leishmaniasis cutánea, lo que conlleva a que los pobladores deban trasladarse hasta el casco urbano, generando un sobrecosto que puede ser mayor si el afectado requiere compañía.

También se detectó que el tipo de vinculación laboral que tiene el personal de laboratorio genera una alta rotación, esto quiere decir que en varios momentos del año encontramos profesionales recién contratados, que no cuentan con la experiencia ni el entrenamiento, dificultando el correcto diagnóstico. Por su parte, los médicos rurales llegan usualmente de otras zonas no endémicas para leishmaniasis cutánea, por lo que durante su formación de pregrado solo tuvieron acercamientos teóricos a la enfermedad. En cuanto al tratamiento, se identificó que se suministra desconociendo los protocolos nacionales e internacionales, lo que podría producir un aumento de eventos adversos o una cura incompleta. 


\section{DISCUSIÓN Y CONCLUSIONES}

Los resultados del presente estudio apuntan hacia algunas conclusiones centrales. Con relación al estudio epidemiológico, se halló una relación entre lo reportado en el SIVIGILA y lo encontrado en las comunidades, pues se evidenció una disminución del 59\% de los casos reportados entre 2014 y 2015, esto podría deberse al fenómeno de El Niño que se presentó en Colombia en el 2015 y generó una disminución de los casos reportados de enfermedades transmitidas por vectores en el territorio nacional(5).

Aunque solo se encontró un caso de leishmaniasis cutánea en la zona durante el periodo del estudio, se pudo demostrar que los pobladores han estado en contacto con el parásito y conocen la enfermedad; sin embargo, la mayoría de ellos aseguran que la leishmaniasis cutánea es más predominante en otras zonas del municipio con áreas más boscosas, como lo reporta la mayoría de participantes con casos antiguos.

El hecho de que la población infantil de la vereda Tuis Tuis no haya presentado casos de leishmaniasis cutánea, nos lleva a pensar en dos causas: inicialmente, que la transmisión no se presenta desde hace algunos años en la zona, o que las prácticas cotidianas de los menores les impide estar en contacto con el vector y sus focos de transmisión. Se destaca que la mayor prevalencia en población adulta se presentó en la vereda El Loro, la cual se encuentra geográficamente más cerca de las zonas inundables de una represa, y es la que cuenta con un mayor número de población desplazada.

La prueba de Montenegro no es concluyente para determinar la prevalencia de la enfermedad en comunidades con población desplazada, ya que este fue un muestreo por conveniencia y no está dentro de los fundamentos de esta técnica identificar infección, sino contacto previo con el parásito, el cual pudo haberse dado en cualquier lugar durante la vida del individuo. Es por esto que se recomienda realizar esta prueba junto a una encuesta epidemiológica que indague sobre los diferentes lugares de residencia de cada individuo, las características ecológicas y sociodemográficas de esos lugares y posibles brotes de la enfermedad, información con la que se podría llegar a hacer una mejor asociación sobre el contacto del individuo con el parásito.

El análisis antropológico muestra que en estas comunidades de Córdoba siempre ha habido leishmaniasis cutánea, según narran los habitantes más antiguos, por ello existe una cultura popular sobre el diagnóstico ${ }^{26}$ y una serie de prácticas terapéuticas tradicionales para hacer frente a la enfermedad. La leishmaniasis cutánea constituye un problema de salud percibida, por ello buscan un tratamiento ${ }^{(19)}$. A pesar de reconocer las lesiones típicas de la leishmaniasis cutánea (grano y Ilaga), los pobladores desconocen el origen de la enfermedad, el vector que la transmite y en qué lugares, cerca o lejos de su vivienda, lo pueden encontrar. Datos similares han sido reportados en otros países como Ecuador, Perú y Costa Rica ${ }^{(6,17,18,19,26)}$.

Las explicaciones que brindan los pobladores respecto a la forma de transmisión de la leishmaniasis cutánea están enmarcadas en las experiencias, propias y ajenas, con otras enfermedades transmitidas por vectores, como la enfermedad de Chagas o la malaria, por ejemplo. Se valen de la reconfiguración de esas experiencias corporales y del saber popular para dar sentido a la enfermedad que los aqueja y de la cual no tienen conocimiento. Por ello encontramos múltiples discursos circulando, explicaciones que distan del conocimiento científico sobre la leishmaniasis cutánea pero que, al interior de las creencias de los pobladores, tienen sentido, dan lugar a la enfermedad en su mundo y, en consecuencia, una solución o cura para ella.

Lo anterior nos permite comprender, por ejemplo, por qué los pobladores no toman medidas de autocuidado frente al vector, ya que dentro del universo simbólico que han construido para la leishmaniasis cutánea no hay una correlación con la Lutzomyia (Alú). La mayoría de los pobladores desconoce el vector y su comportamiento (lugares y horas 
en que pica); además, la interacción permanente con diferentes insectos ha hecho que se "familiaricen" con ellos y solo tomen medidas de prevención cuando hay un aumento de la masa de mosquitos. Sumado a que no hay una percepción de riesgo, ya que cuando se toman medidas de protección, es para evitar molestias como las picaduras, pero no porque perciban en el Alú un riesgo potencial de enfermedad.

En cuanto a los tratamientos, hay una clara preferencia por los tradicionales caseros $^{(19,26,27)}$, puesto que resultan ser la mejor opción para comunidades rurales alejadas, con acceso limitado a los servicios médicos de primer nivel, a pesar de que las Secretarías de Salud brindan el tratamiento con Glucantime ${ }^{\circledR}$ de forma gratuita ${ }^{(17)}$. Entre los tratamientos tradicionales se destaca el uso de plantas medicinales, tal como ocurre en otras zonas de Colombia y en países vecinos como Ecuador o Perú(26). Estos tratamientos son principalmente brindados por las mujeres del hogar, por lo que que ellas deberían ser un actor clave en los procesos de educación con comunidades, más aún cuando algunos de los tratamientos empíricos han empeorado el cuadro clínico ${ }^{(17)}$, porque aumentan la inflamación en la zona de la herida, se produce necrosis y se alteran las lesiones típicas, generando, incluso, disminución de sensibilidad del diagnóstico parasitológico(26).

Es importante señalar que el desconocimiento que tiene el personal de salud local frente a los protocolos nacionales e internacionales ha generado eventos adversos durante algunos tratamientos, lo que acrecienta la respuesta negativa de las comunidades frente al tratamiento biomédico, así como la falta de credibilidad en las autoridades de salud $y$, en consecuencia, perciben como mejor la atención del personal que ejerce la medicina tradicional.

En cuanto a los actores clave dentro de las comunidades, encontramos que uno de los resultados más interesantes es la estrecha similitud entre las concepciones de los docentes, las personas en general y los líderes comunitarios en salud. En los tres grupos encontramos desconocimiento sobre la causa de la enfermedad y la transmisión, así como percepciones negativas frente al tratamiento biomédico versus tratamientos de la medicina popular.

La mayoría de los docentes lograban explicar mucho mejor la leishmaniasis cutánea desde el campo de las creencias, que desde el conocimiento científico y, en algunos casos, reproducían prácticas de riesgo. Respecto de los líderes en salud, han sido capacitados en el diagnóstico, el tratamiento y la prevención de la malaria, arrojando resultados sobresalientes. Sin embargo, y pese a estar en una zona endémica, no han sido capacitados sobre leishmaniasis cutánea, por lo que encontramos que no hay mayor diferencia entre el saber popular de los demás habitantes y los líderes en salud respecto a la leishmaniasis cutánea, lo que pone de manifiesto que la comunicación y validación del saber popular por parte de las autoridades de salud es importante para poder generar campañas de prevención efectivas que estén direccionadas al contexto rural ${ }^{(27)}$.

Por último, es relevante señalar que la disminución en los últimos dos años de casos de leishmaniasis cutánea, reportada por los habitantes de las cuatro comunidades del estudio, sugiere que la gran mayoría de los 127 casos registrados en Tierralta por el Sistema Nacional de Vigilancia SIVIGILA podrían ser de pacientes de las Fuerzas Armadas Colombianas, que patrullan en la Serranía de Abibe. Esto sugeriría que la infección y ciclo natural de la enfermedad está ocurriendo en zonas alejadas y selváticas, donde la mayoría de las autoridades de salud no tienen acceso, ya que son de difícil ingreso y con condiciones de orden público complejas.

\section{RECOMENDACIONES}

Conscientes de los retos de las intervenciones en salud, generamos cuatro recomendaciones en aras de disminuir la brecha de acceso a la atención para las personas que viven en zonas endémicas para leishmaniasis cutánea. 
El primer reto consiste en involucrar efectivamente al sector educativo. En estos contextos vulnerables, en los que el nivel educativo de sus habitantes es bajo, el docente sobresale como un referente del "saber", un actor clave al que las personas consultan y piden información, pero encontramos que tienen vacíos frente a la leishmaniasis cutánea. Con este panorama se recomienda implementar procesos formativos con docentes sobre leishmaniasis cutánea, para cambiar potencialmente este escenario ${ }^{(27)}$ puesto que educar a partir de estrategias que surjan de las concepciones locales sobre la enfermedad y que posibiliten transformar imaginarios podría significar la incorporación de nuevo conocimiento y la adopción de prácticas de autocuidado al interior de las comunidades.

El segundo reto radica en aprovechar el recurso humano propio de las comunidades. Actualmente, los líderes comunitarios de la zona rural son un recurso desaprovechado por las instituciones locales de salud, si consideramos que ellos son los principales receptores de casos emergentes en las veredas. Las estrategias de intervención en salud deben incluir a los líderes, apalancarlos en su rol activo y direccionarlos con miras a dejar capacidad instalada dentro de las comunidades.

El tercer reto es una apuesta a la capacitación y actualización sobre leishmaniasis cutánea al personal de salud. Es apremiante el manejo de las guías nacionales e internacionales, ya que los fallecimientos asociados a la leishmaniasis cutánea son precisamente por el tratamiento con Glucantime ${ }^{\circledR}$ : un mal manejo puede desencadenar pérdidas prevenibles. Se requiere de personal capacitado que maneje un flujo correcto de información entre los diferentes sistemas terapéuticos, que oriente a los pacientes para un adecuado diagnóstico y tratamiento, además de fortalecer los programas y estrategias de prevención $^{(17,27)}$.

El cuarto reto consiste en un acercamiento entre el personal de salud y el sistema médico local. La medicina tradicional debe empezar a cobrar un papel importante en el tratamiento de la leishmaniasis cutánea. Es necesario que el personal de salud conozca los tratamientos locales y sepa orientar a sus pacientes cuando recurren a ellos, pues los casos ocurren lejos de los cascos urbanos, lo que propicia que los pacientes hagan uso de plantas y otros elementos como primera línea terapéutica.

Finalmente, es imperante agregar que se requiere un manejo integral de la leishmaniasis cutánea, que incluya la participación de profesionales de diferentes áreas para intervenir sobre todas las dimensiones de la enfermedad. Se destaca el trabajo del componente social, porque facilita el diálogo entre los pobladores y la comunidad científica, con miras a generar soluciones reales basadas en las necesidades particulares de cada comunidad.

\section{AGRADECIMIENTOS}

Este artículo se deriva del proyecto de investigación "Diseño de un programa de estudios en infecciones y salud tropical para el departamento de Córdoba", financiado por la Gobernación de Córdoba y el Sistema General de Regalías de Colombia (BPIN 2012000100176) y desarrollado por la Universidad del Sinú, en convenio con el Programa de Estudio y Control de Enfermedades Tropicales (PECET) de la Universidad de Antioquia.
Queremos agradecer a las cuatro comunidades del estudio de los municipios de Tierralta y Valencia, a sus líderes comunitarios, maestros y personal de salud por abrirnos las puertas y permitirnos compartir sus conocimientos. A todo el equipo asistente del laboratorio del PECET de la Universidad de Antioquia y del laboratorio de Investigaciones Biomédicas de la Universidad del Sinú, especialmente a Jorge Negret, Horacio Cadena y Andrés Felipe Vélez por toda su colaboración. 


\section{REFERENCIAS BIBLIOGRÁFICAS}

1. Isaza DM, Restrepo BN, Arboleda M, Casas E, Hinestroza $H$, Yurgaqui T. La leishmaniasis: conocimientos y prácticas en poblaciones de la costa del Pacífico de Colombia. Revista Panamericana de Salud Pública. 1999;6(3):177-184.

2. Organización Mundial de la Salud. Leishmanisis: Nota descriptiva N³75 [Internet]. 2016 [citado 1 jul 2016]. Disponible en: http://tinyurl. com/hz32wy4.

3. Ministerio de la Protección Social, Instituto Nacional de Salud, Organización Panamericana de la Salud. Guía para la atención clínica integral del paciente con leishmaniasis [Internet]. Bogotá: Ministerio de la Protección Social; 2010 [citado 1 jul 2016]. Disponible en: http://tinyurl.com/z5rnf5z.

4. Instituto Nacional de Salud, Dirección de Vigilancia y Análisis del Riesgo en Salud Pública. Semana epidemiológica número 51 de 2014. Boletín Epidemiológico Semanal [Internet]. 2014 [5 jul 2016]. Disponible en: http://tinyurl.com/ gnwwrev.

5. Instituto Nacional de Salud, Dirección de Vigilancia y Análisis del Riesgo en Salud Pública. Semana epidemiológica número 52 de 2015. Boletín Epidemiológico [Internet]. 2015 [5 jul 2016]. Disponible en: http://tinyurl.com/z8pq4bj.

6. Dobles-Ulloa A, Perriard C. Representaciones, actitudes y prácticas respecto a la leishmaniasis cutánea en la población del Cantón de Acosta, provincia de San José, Costa Rica: estudio antropológico exploratorio. Cadernos de Saúde Pública. 1994;10(2):181-189.

7. Organización Mundial de la Salud. Acción de la Organización Mundial de la Salud en el Afganistán para combatir la leishmaniasis debilitante [Internet]. 10 ago 2014 [citado 18 jun 2016]. Disponible en: http://tinyurl.com/mjet9ku.

8. Díaz Díaz B. Sociología de la leishmaniasis en Trujillo, área andina de Venezuela. Mérida: Universidad de Los Andes; 2014.

9. Vélez ID, Hendrickx E, Robledo SM, del Pilar Agudelo S. Leishmaniosis cutánea en Colombia y género. Cadernos de Saúde Pública. 2001;17(1):171-180.

10. Carrillo-Bonilla LM, Trujillo JJ, Álvarez-Salas L, Vélez-Bernal ID. Estudio de los conocimientos, actitudes y prácticas de la leishmaniasis: evidencias del olvido estatal en el Darién Colombiano. Cadernos de Saúde Pública. 2014;30(10):2134-2144.
11. Colombia. Resultados del Censo General 2005: Población censada después de compensada por omisiones de cobertura geográfica $(1,22 \%)$ y contingencia de transferencia $(0,26 \%)$ [Internet]. Bogotá: Departamento Administrativo Nacional de Estadística; 2005 [citado 13 de enero de 2017]. Disponible en: http://tinyurl.com/k735f6n.

12. Holdridge L. Life zone ecology. San José: Tropical Science Center; 1967.

13. Pifano F, Romero M. Investigaciones epidemiológicas sobre la leishmaniasis visceral en la Isla de Margarita Estado Nueva Esparta, Venezuela. Gaceta Médica de Caracas. 1964;(72):425-430.

14. Pifano F, Romero M. Comprobación de un foco autóctono de leishmaniasis visceral (kala-azar) en la Isla de Margarita, Estado Nueva Esparta, Venezuela. Archivos Venezolanos de Medicina Tropical y Parasitología Médica. 1973;(5):134-144.

15. Delgado O, Feliciangeli M, Gómez R, Álvarez J, García L, Bello C. The re-emergence of American Visceral Leishmaniasis in an old focus in Venezuela: Present situation on human and canine infections. Parasite. 1998;(5):317-323.

16. Valencia Jiménez NN, Solera Martínez NF. Necesidades de aprendizaje para la sexualidad de jóvenes entre 10 y 19 años en el caribe colombiano. Investigación y Desarrollo. Investigación y Desarrollo. 2009;17(1):106-131.

17. Vélez ID. Leishmaniosis en Colombia: concepciones, actitudes y prácticas en comunidades indígenas y campesinas. En: Actas del Tercer Congreso Chileno de Antropología. Temuco: Colegio de Antropólogos de Chile; 1998. p. 130-137.

18. Trujillo J, Carrillo L, Vélez I. Estudio de las concepciones, actitudes y prácticas de la leishmaniosis en dos regiones del país, el primer paso para contribuir a su control. Biomédica. 2009;29(Supl. 1):58-64.

19. Gómez L, Corredor A. Caracterización sociocultural y epidemiológica de un foco de leishmaniasis cutánea en Cimitarra, Santander. Revista de Salud Pública. 2000;2(3):261-271.

20. Corbin J, Strauss A. Bases de la investigación cualitativa, técnicas y procedimientos para desarrollar la teoría fundamentada. Medellín: Editorial Universidad de Antioquia; 2002.

21. Kemp M, Hey AS, Kurtzhals JA, Christensen CB, Gaafar A, Mustafa MD, Kordofani AA, Ismail A, Kharazmi A, Theander TG. Dichotomy of the human $\mathrm{T}$ cell response to Leishmania antigens: I Th1-like response to Leishmania major promas- 
tigote antigens in individuals recovered from cutaneous leishmaniasis. Clinical \& Experimental Immunology. 1994;96(3):410-415.

22. Weigle $K$, Valderrama L, Arias AL, Santrich C, Saravia NG. Leishmanin skin test standardization and evaluation of safety, dose, storage, longevity of reaction and sensitization. American Journal of Tropical Medicine and Hygiene. 1991;44(3):260-271.

23. Vélez Bernal ID, Robledo Restrepo SM. Vacuna contra la leishmaniasis: Descubrimiento y ensayos clínicos. En: Corredor Pereira C, Guhl Nannetti F, Duque Beltrán C. Tendencias y futuros de la investigación en parasitología y en productos naturales. Bogotá: ACOFACIEN, ACCEFYN; 2009. p. 63-73.

24. Fraga J, Montalvo AM, De Doncker S, Dujardin JC, Van der Auwera G. Phylogeny of Leishmania species based on the heat-shock protein 70 gene. Infection, Genetics and Evolution. 2010;10(2):238-245.
25. Fernández Z, Oviedo M, Vívenes M, Maffei M, González A, Vásquez L. Leishmaniasis visceral en Trujillo, Venezuela: conocimientos, actitudes, prácticas (CAP) y estrategias de prevención y control. Fermentum. 2011;21(60):45-64.

26. Pineda-Reyes R, Llanos-Cuentas A, Dancuart M. Tratamientos tradicionales utilizados en un área endémica de leishmaniasis cutánea en el Perú. Revista Peruana de Medicina Experimental y Salud Pública. 2015;32(4):761-765.

27. Vázquez $M$, Kroeger A, Lipowsky R, Alzate A. Conceptos populares sobre la leishmaniasis cutánea en Colombia y su aplicabilidad en programas de control. Boletín de la Oficina Sanitaria Panamericana. 1991;110(5):402-415.

\section{FORMA DE CITAR}

Patiño Londoño SY, Salazar LM, Tovar Acero C, Vélez Bernal ID. Aspectos socioepidemiológicos y culturales de

la leishmaniasis cutánea: concepciones, actitudes y prácticas en las poblaciones de Tierralta y Valencia, (Córdoba, Colombia). Salud Colectiva. 2017;13(1):123-138. doi: 10.18294/sc.2017.1079.

Recibido: 28 de julio de 2016 | Versión final: 14 de febrero de 2017 | Aprobado: 8 de marzo de 2017

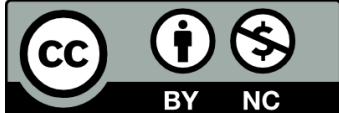

Este obra está bajo una licencia de Creative Commons Reconocimiento-NoComercial 4.0 Internacional. Reconocimiento - Permite copiar, distribuir y comunicar públicamente la obra. A cambio, se debe reconocer y citar al autor original. No Comercial - Esta obra no puede ser utilizada con finalidades comerciales, a menos que se obtenga el permiso. 